\title{
Transcranial direct current stimulation of the prefrontal cortex modulates working memory performance: combined behavioural and electrophysiological evidence
}

\author{
Tino Zaehle $e^{1,2^{*} \dagger}$, Pascale Sandmann ${ }^{3 \dagger}$, Jeremy D Thorne ${ }^{3}$, Lutz Jäncke ${ }^{4}$, Christoph S Herrmann ${ }^{5}$
}

\begin{abstract}
Background: Transcranial direct current stimulation (tDCS) is a technique that can systematically modify behaviour by inducing changes in the underlying brain function. In order to better understand the neuromodulatory effect of tDCS, the present study examined the impact of tDCS on performance in a working memory (WM) task and its underlying neural activity. In two experimental sessions, participants performed a letter two-back WM task after sham and either anodal or cathodal tDCS over the left dorsolateral prefrontal cortex (DLPFC).

Results: Results showed that tDCS modulated WM performance by altering the underlying oscillatory brain activity in a polarity-specific way. We observed an increase in WM performance and amplified oscillatory power in the theta and alpha bands after anodal tDCS whereas cathodal tDCS interfered with WM performance and decreased oscillatory power in the theta and alpha bands under posterior electrode sides.

Conclusions: The present study demonstrates that tDCS can alter WM performance by modulating the underlying neural oscillations. This result can be considered an important step towards a better understanding of the mechanisms involved in tDCS-induced modulations of WM performance, which is of particular importance, given the proposal to use electrical brain stimulation for the therapeutic treatment of memory deficits in clinical settings.
\end{abstract}

\section{Background}

Transcranial direct current stimulation (tDCS) is a technique that stimulates the cerebral cortex with a weak constant electric current in a non-invasive and painless manner [1]. The current flows from an active to a reference electrode, a part being shunted through the scalp and the rest being delivered to the brain tissue [2], thereby inducing diminutions or enhancements of cortical excitability [1]. The direction of the tDCS-induced effect depends on the current polarity: Anodal tDCS typically has an excitatory effect on the local cerebral cortex, while cathodal tDCS decreases the cortical excitability in the region under the electrode $[3,4]$. The mechanisms underlying these neuromodulatory effects are not well understood [5]. Animal studies suggest that

\footnotetext{
* Correspondence: tino.zaehle@DZNE.de

+ Contributed equally

'Department of Neurology, Otto v. Guericke University Magdeburg, Germany Full list of author information is available at the end of the article
}

anodal tDCS, via an extracellular negative sink, causes a depolarization of the resting-membrane potential and increases the firing rates of many perpendicularly oriented cortical neurons in the tissue under the electrode. Cathodal stimulation has the opposite effect, causing a hyperpolarisation of the resting-membrane potential and a decrease in firing rates [6,7]. Thus, tDCS seems to modify spontaneous neural excitability by tonic de- or hyperpolarization of the resting-membrane potential [1]. However, the effects of tDCS are not limited to modulations in cortical excitability during stimulation, and may outlast the stimulation period by several minutes or even hours $[3,4,6,8]$. These after effects of tDCS are associated with a number of different mechanisms, including local changes in ionic concentrations (hydrogen, calcium) and levels of cyclic adenosine monophosphate (cAMP), alterations in protein synthesis, and modulation of $\mathrm{N}$-methyl-D-aspartate (NMDA) receptor efficacy [5,9-14]. 
The neuromodulatory changes induced by tDCS have been associated with modifications of a variety of behavioural brain functions. In animal studies, anodal tDCS of the cortical surface has been linked with facilitation of an unconditioned response $[15,16]$ and improved learning [17]. In humans, the effects of tDCS have been demonstrated on various motor, visual, and somatosensory cortex functions (see [18] for a recent review). In particular, previous studies have reported enhancements in motor $[19,20]$ and visuo-motor learning [21] for anodal tDCS, while impairments in auditory learning have been observed for cathodal tDCS [22]. Similarly, anodal tDCS improves language learning [23], picture-naming [24] as well as implicit grammar learning [25], whereas cathodal tDCS has been shown to impair verbal learning abilities [26,27]. Analogous polarity-specific effects of tDCS have been reported for working memory (WM) functions, suggesting that anodal but not cathodal tDCS can improve WM performance $[8,28,29]$. However, the nature of the neurophysiological mechanisms underlying this cognitive enhancement is not yet well understood, because modifications of WM functions by tDCS have never been studied in combination with neurophysiological methods.

In general, WM refers to a set of basic mental operations that define the ability to hold an item of information transiently in mind, in order to recall, manipulate and associate this information to incoming new information [30]. WM is crucial to many higher-order strategic functions and has been linked to frontal [31,32] and parietal lobe functioning [33]. A commonly used WM paradigm is the $\mathrm{n}$-back task which activates a frontoparietal network, including the dorsolateral prefrontal cortex (DLPFC) [34-37], and the posterior parietal cortex [38]. While the DLPFC is involved in the processing of stimulus information during retention times [39], the parietal lobe participates in the storage of perceptual attributes [40]. Furthermore, the prefrontal cortex seems to be functionally lateralized, with the right hemisphere being recruited in particular during spatial WM tasks, and the left hemisphere being crucial for the processing of non-spatial (i.e., verbal) WM tasks [41]. The critical role for the left DLPFC in verbal WM performance has been confirmed by lesion studies and studies using TMS, showing that focal damage and temporary disruption of the left but not the right DLPFC is related to impairments in verbal WM task performance $[42,43]$.

The present study examined the impact of tDCS on WM performance and the underlying neural activity. In particular, we explored the effect of tDCS applied over the left DLPFC on oscillatory brain activity during a letter n-back WM task. Based on previous findings we hypothesized tDCS-dependent alteration of WM performance $[8,29]$. Furthermore, we predicted tDCS-related modifications of the underlying rhythmic neural activity in the alpha and theta frequency range, given the view that alpha and theta oscillations play an important role in memory functions [44]. To our knowledge this is the first study investigating the modulatory effects of tDCS on oscillatory brain activity in the context of a WM task. The better understanding of the neuromodulatory effects of tDCS is also of clinical interest, since electrical brain stimulation seems to have potential as a therapeutic tool applied for several neurological and psychiatric disorders [45-51], and particularly for the treatment of memory deficits in stroke patients [52], patients with Parkinson's disease [28], and patients suffering from Alzheimer's disease [53,54].

\section{Results}

\section{Behavioural data}

Pre-to-post measurements revealed that all participants improved their performance during the experiment. Table 1 shows the results of one-sample t-tests separately for the tDCS-treated group in each stimulation condition (delta anodal $\Delta \mathrm{A}$, delta cathodal $\Delta \mathrm{C}$ ) and for the control group (delta $\Delta C G$ ). The behavioural improvement from sham to active tDCS was stronger after anodal than after cathodal stimulation (cf. Figure 1). This polarity effect was statistically significant for the comparison of $\Delta \mathrm{A}-\mathrm{d}^{\prime}>\Delta \mathrm{C}-\mathrm{d}^{\prime}\left(t_{15}=2.14, P<0.05\right)$. Analysis of reaction times (RT) showed that tDCS-treated participants responded faster after active tDCS compared to sham for Hits, but not for False Alarms (cf. Table 1). Similarly, pre-to-post measurements for the control group showed faster RT for Hits but not for False Alarms. Interestingly, the decrease of RT in the tDCS-treated group did not differ between anodal and cathodal stimulation $(\Delta \mathrm{A}=\Delta \mathrm{C})$, neither for Hits $\left(t_{15}=\right.$ $0.11, P=0.9)$ nor for False Alarms $\left(t_{15}=-0.13, P=0.9\right)$.

In order to separate tDCS-induced alterations of WM performance from repetition-related learning effects, we

Table 1 Improvement in performance

\begin{tabular}{lllll}
\hline condition & measure & $\boldsymbol{t}$ & df & $\boldsymbol{P}$ \\
\hline $\boldsymbol{\Delta A}$ & $\mathrm{d}^{\prime}$ & 4.748 & 15 & 0.000 \\
& RT-Hits & -5.370 & 15 & 0.000 \\
& RT-FA & -1.210 & 15 & 0.244 \\
$\boldsymbol{\Delta C}$ & d' & 2.751 & 15 & 0.015 \\
& RT-Hits & -3.760 & 15 & 0.002 \\
& RT-FA & -0.945 & 15 & 0.359 \\
$\boldsymbol{\Delta C G}$ & d' & 3.944 & 15 & 0.001 \\
& RT-Hits & -4.289 & 15 & 0.001 \\
& RT-FA & -0.600 & 15 & 0.555 \\
\hline
\end{tabular}

Table lists result of one-sample t-tests for d', RT-Hits (reaction time for Hits), and RT-FA (reaction time for False Alarms). Results show a general

improvement in behaviour from pre- to post measurements for anodal $(\triangle A)$ and cathodal stimulation $(\Delta C)$, and in the separate control group $(\Delta C G)$. 

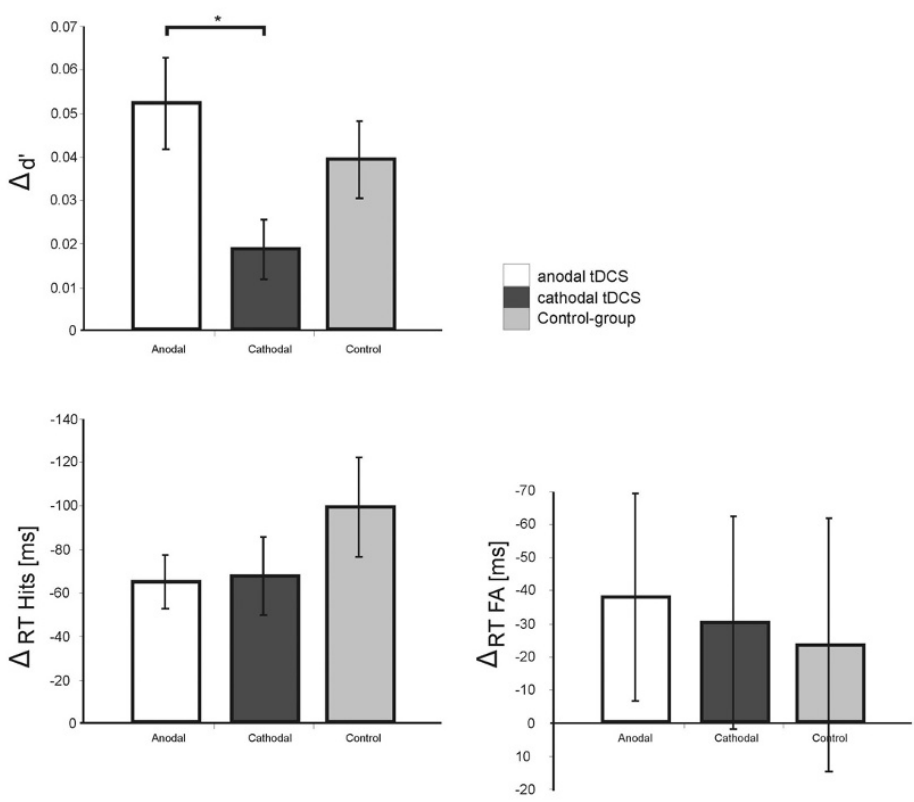

Figure 1 Behavioural Data: Plots show performance in the WM task after anodal and cathodal tDCS in relation to preceding sham stimulation as well as the performance of the separate control group. Top: The $d^{\prime}$ increased more after anodal than after cathodal stimulation with an intermediate effect for the control group, demonstrating a polarity effect of the tDCS-induced behavioural improvement. Bottom: Participants responded generally faster from sham to tDCS measure and pre-to-post measure in the control group for Hits (left) and False Alarms (right), respectively. Asterisks indicate statistical significance $(P<0.05)$.

compared the behavioural pre-to-post measurements between the tDCS-treated group and the control group (cf. Figure 1). The comparison revealed for the tDCStreated group a stronger, but statistically non-significant d' increase after anodal stimulation $\left(\Delta \mathrm{A}-\mathrm{d}\right.$ ' $>\Delta$ CG-d', $t_{30}$ $=0.99, P=0.33)$, and a weaker $\mathrm{d}^{\prime}$ increase after cathodal stimulation $\left(\Delta \mathrm{C}-\mathrm{d}^{\prime}<\Delta \mathrm{CG}-\mathrm{d}^{\prime}, t_{30}=-1.39, P=0.17\right)$ compared to the control group. Analysis of RT showed that pre to post decreases did not differ between the control group and the tDCS-treated group for the anodal or cathodal session, either for Hits $\left(\Delta \mathrm{A}=\Delta \mathrm{CG}: t_{30}=1.29\right.$, $\left.P=0.2, \Delta \mathrm{C}=\Delta \mathrm{CG}: t_{30}=1.1, P=0.3\right)$ or for False Alarms $\left(\Delta \mathrm{A}=\Delta \mathrm{CG}: t_{30}=-0.3, P=0.8, \Delta \mathrm{C}=\Delta \mathrm{CG}: t_{30}\right.$ $=-0.14, P=0.9)$.

\section{Event-related potentials (ERPs)}

Figure 2 illustrates the event-related potential (ERP) data for anodal, cathodal and the two sham conditions (shamA, shamC) averaged over 16 subjects for the occipito-parietal region of interest (ROI) and electrode Pz. Visual stimulation consistently evoked a P1 component at $118 \mathrm{~ms}$ which was followed by the N1 component at 174 ms. A P3 component was elicited consistently in all tDCS conditions with a mean latency of 336 ms. Paired t-tests were performed to statistically compare the active and corresponding sham conditions (anodal vs. shamA, cathodal vs. shamC), and the anodal and cathodal conditions (shamA vs. shamC, anodal vs. cathodal). Statistical analyses of ERP amplitudes revealed no significant differences between conditions for the P1, N1 and P3 components. Regarding latencies, no significant differences were found between the two sham conditions. However, N1 latencies were significantly longer for the anodal than for the cathodal $\left(t_{15}=2.58, P<0.05\right)$ and for the anodal compared to the shamA condition $\left(t_{15}=-2.28\right.$, $P<0.05)$, and $P 3$ latencies were significantly shorter for the cathodal compared to the shamC condition $\left(t_{15}=\right.$ 2.24, $P<0.05)$.

\section{Event-related spectral perturbation (ERSP)}

Figure 3 shows the event-related spectral perturbation (ERSP) time-frequency plots for the sham conditions (shamA, shamC) and the active stimulation conditions (anodal, cathodal), plus the corresponding differences at the occipito-parietal ROI. Statistical comparison of ERSPs between the shamC and cathodal stimulation condition revealed a decrease in the theta and alpha band $(5-15 \mathrm{~Hz})$, at the latency range of $14-710 \mathrm{~ms}$ $\left(t_{15} \geq 2.13, P<0.05\right)$. Anodal tDCS as compared to cathodal tDCS increased the total power in the theta, 

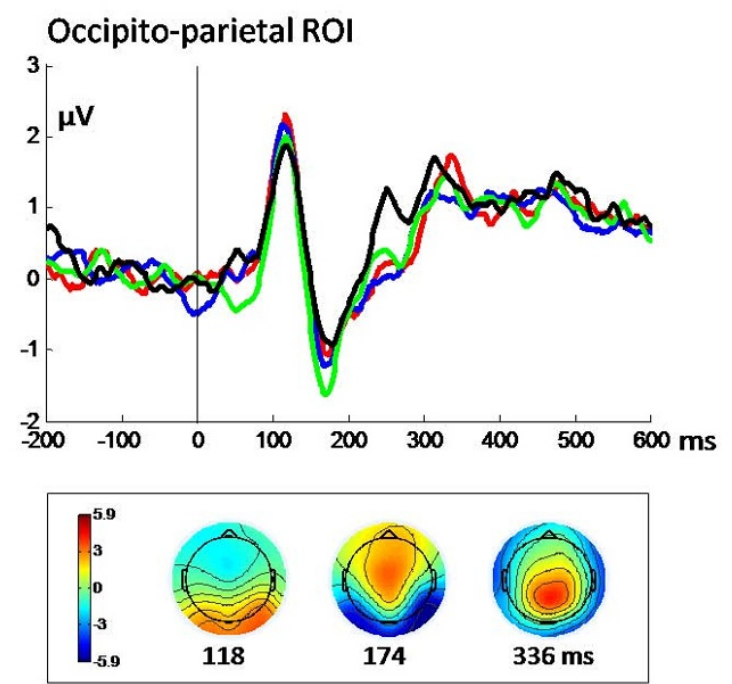

Channel Pz

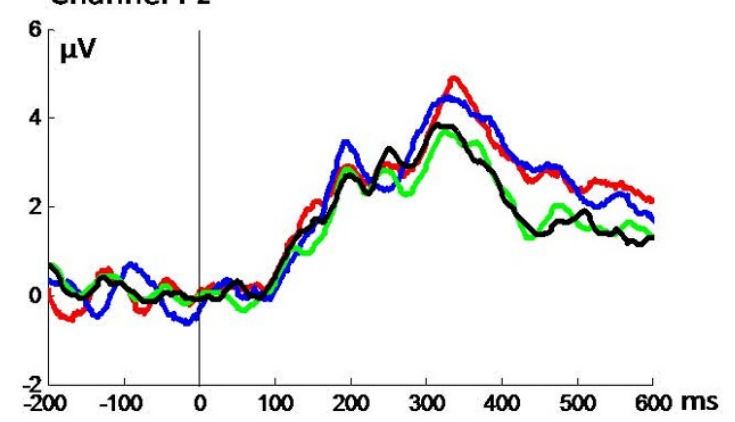

Figure 2 Event-related potentials: Grand average event-related potentials (ERPs) are shown for each condition at the occipitoparietal ROI (above) and at channel Pz (below). Topographies of overall grand averages (computed across all participants and conditions) are shown at P1, N1, and P3 latencies.

alpha, and lower beta band (7-24 Hz) at the latency range of $-72-380 \mathrm{~ms}\left(t_{15} \geq 2.13, P<0.05\right)$. Finally, a significant cluster $\left(t_{15} \geq 2.13, P<0.05\right)$ was found for the overall differences between sham and stimulation conditions ((anodal - shamA) vs. (cathodal - shamC)). This cluster had a latency range of -34 - $710 \mathrm{~ms}$ and revealed a significant increase in the theta, alpha, and lower beta band $(6-19 \mathrm{~Hz})$ for the anodal (anodal shamA) compared to the cathodal session (cathodal shamC).

\section{Discussion}

We assessed the impact of transcranial direct current stimulation (tDCS) on working memory (WM) performance and the underlying neural activity. In two experimental sessions participants performed a letter two-back WM task after sham and either anodal or cathodal tDCS. The results showed that WM performance was generally enhanced from the sham to the active stimulation condition, indicating a repetition-related learning effect on WM task performance. Importantly, this regular improvement in WM performance was influenced by active $\mathrm{tDCS}$ in a polarity-specific way. The improvement in WM performance was significantly stronger after the application of anodal than after cathodal tDCS over the left dorsolateral prefrontal cortex (DLPFC), while the WM improvement was intermediate when participants received no electrical stimulation. Thus, anodal tDCS improved the regular repetition-related increase in WM performance, whereas cathodal tDCS interfered with this effect. These tDCS-induced effects were reflected in the neural oscillatory activity, showing polarity-specific alterations as a function of tDCS. Anodal tDCS increased whereas cathodal tDCS decreased the event-related oscillatory power in the theta and alpha range. Our results suggest that tDCS altered WM performance by modulating the underlying oscillatory brain activity in the theta and alpha frequency bands. These results we consider an important step towards a better understanding of the mechanisms involved in the tDCS-induced modulations of WM performance, which is particularly relevant as electrical brain stimulation has been proposed as a useful therapeutic modality for the treatment of memory deficits in a clinical context $[28,52]$. More specifically, the present results of tDCSinduced modulations of neural oscillations in specific frequency bands suggests that the combination of tDCS and EEG might provide a useful approach for the investigation of the functional significance of several oscillatory bands in human cognition. Furthermore, our results may provide additional explanations for tDCSrelated therapeutic effects in patients suffering from Parkinson's disease or Alzheimer' disease. Based on our findings we predict that beneficial effects of tDCS in these patients may be associated with modulations in oscillatory brain activity, in particular in the alpha and theta frequency range. In addition, our results may inspire future research on tDCS-related therapeutic applications, in particular with respect to pathologies that have been associated with alterations of oscillatory brain activity in specific frequency bands.

\section{Behavioural effects of tDCS}

The effect of tDCS on WM performance is consistent with recent findings on the modulatory effects of tDCS on WM functions $[8,28,29,52]$. Similar to our results, anodal but not cathodal stimulation over the left DLPFC has been shown to increase performance in a sequential-letter WM task [8,29]. Likewise, a beneficial effect of anodal tDCS over the left DLPFC on WM has been observed in patients with Parkinson's disease [28], and in patients suffering from stroke [52]. Our study further 


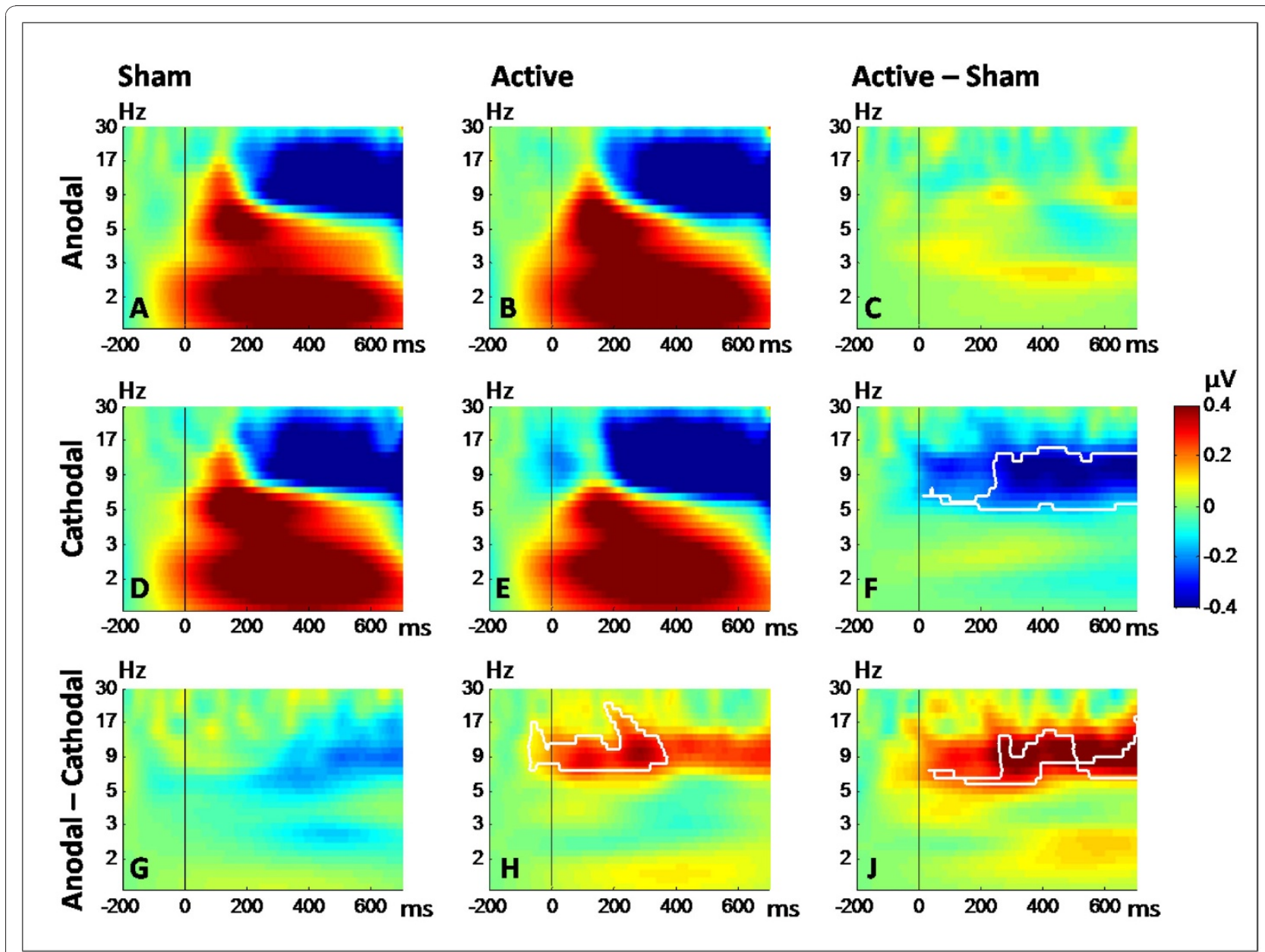

Figure 3 DC stimulation effect on oscillatory brain activity: Event-related spectral perturbation (ERSP) time-frequency plots are given separately for the two sham conditions (A: sham anodal; D: sham cathodal) preceding the two active stimulation conditions (B: anodal, E: cathodal). Differences between the conditions were computed by subtracting the sham ERSPs from the ERSPs of the active conditions (C: active anodal - sham anodal; F: active cathodal - sham cathodal) and by subtracting the cathodal ERSPs from anodal ERSPs (G: sham anodal - sham cathodal; $\mathrm{H}$ : active anodal - active cathodal). Subplot J illustrates the overall contrast between the differences obtained for the anodal and cathodal conditions (subplot $\mathrm{C}$ - subplot F) and for the active and sham conditions (subplot $\mathrm{G}$ - subplot H). White contours indicate significant differences between the conditions ( $P<0.05$, corrected for multiple comparisons). Note that the statistical analyses revealed no significant differences between the two sham conditions.

revealed that the general decrease in response time (RT) from sham to active tDCS did not vary with tDCS polarity. Similar RT insensitivity to tDCS has been previously reported $[8,28,29,52]$, although one former study has observed increased RT after both anodal and cathodal stimulation over fronto-cortical regions [55]. This discrepancy between behavioural findings might be explained by the fact that the latter study evaluated the effects of bilateral and intermittent tDCS, while our study investigated unilateral and continuous tDCS. Furthermore, the difference in results between the studies may be a consequence of differences in electrode size, position and applied current intensities, and duration of the washout period between the active tDCS sessions. Computational approaches using spherical [2,56] and realistic finite element head models [57] have demonstrated that the size, location and shape of the stimulating electrode can influence the electric field in the underlying brain, thereby affecting the level of modulation of tDCS. Overall, our behavioural results, even though not novel per se, provide further evidence for a modulatory capability of tDCS on WM performance. Our results confirm previous findings by showing that tDCS can reliably induce alterations in WM performance in a polarity-dependent manner. More specifically, our results suggest that repetition-related improvement in WM performance is elevated by anodal tDCS, but diminished by cathodal tDCS. 


\section{Electrophysiological correlates of tDCS effects}

In this study we assessed oscillatory brain activity during the WM task in order to investigate the underlying neural mechanisms mediating the tDCS-induced behavioural effects. To date, reports of electrophysiological correlates of tDCS effects are sparse. Using visual eventrelated potentials (ERPs), a previous study has demonstrated that the amplitude of the N70 ERP component is increased by anodal tDCS, while it is decreased by cathodal tDCS [58]. The opposite effect has been reported for the visual P100, showing reduced amplitudes for anodal and increased amplitudes for cathodal stimulation [59]. Polarity-specific changes have also been observed for ERPs in the somatosensory modality [60-62], and for motor cortex excitability [63]. Furthermore, previous studies have shown that cathodal tDCS over the visual cortex decreases beta and gamma activity in response to visual stimulation [64], whereas cathodal tDCS over the motor cortex increases resting state theta and delta oscillation [5]. However, the study of oscillatory brain activity in the context of tDCS-induced WM alterations addresses a research question that has been under-investigated so far. Given that electrical brain stimulation can be a useful therapeutic tool for the treatment of cognitive deficits [65], a better understanding of tDCS-induced effects on the underlying brain activity seems to be of clinical relevance. In the present study we show that tDCS over the left DLPFC modulates theta and alpha band activity during WM performance in a polarity-specific way. Oscillatory power in the theta and alpha range was increased after anodal stimulation, while it was decreased after cathodal tDCS.

\section{Oscillatory brain activity in the context of a WM task}

Changes in oscillatory brain activity play an important role in the formation of perception and memory and thus are essential for higher cognitive functions $[66,67]$. Accordingly, WM representations seem to be sustained by oscillatory brain activity $[68,69]$. Indeed, WM operations have been related to oscillatory brain activity in multiple frequency bands, including the theta $(4-8 \mathrm{~Hz})$, alpha $(8-12 \mathrm{~Hz})$, and beta $(12-30 \mathrm{~Hz})$ range [44]. In particular the performance in visual n-back tasks has been specifically associated with alterations in event-related theta and alpha band activity [70]. In this regard alpha band activity is assumed to reflect a general inhibition of non-task relevant areas [71,72] and may index the degree of inhibition necessary during internally, as opposed to externally, directed attention [73]. In contrast, theta band activity has been associated with memory encoding and retrieval [74-77] and may thus be particularly related to the function of the central executive of the WM system [78-80]. In the context of a WM task, theta band activity seems to reflect the continuous maintenance and manipulation of information required during the performance of an n-back task [70]. Interestingly, a power increase has been previously shown for both the alpha and theta band as a function of practice in a WM task [81].

In sum, previous studies have shown that alterations of rhythmic activity, specifically in the theta and alpha range, are associated with proper WM performance. These oscillations increase with behavioural enhancement. In the present study we show that tDCS over the left DLPFC induces altered WM performance by modulating its underlying theta and alpha activity. This result further highlights the importance of the left DLPFC and the specific role of theta and alpha activity during WM performance. Even though this interpretation implies that the modulatory effects of tDCS on WM are specifically related to the responsiveness of the left DLPFC, it may be assumed that altered local cortical excitability in one part of the responsible network influences the whole neural network associated with WM functions beyond the site of stimulation. Indeed, widespread tDCS-induced changes in cortical activity have been demonstrated by a previous neuroimaging study [82] Thus, it is likely that by influencing one component of the WM network, the electrical stimulation of the left DLPFC had an influence on the functioning of the entire WM system.

\section{Conclusions}

The present investigation studied the impact of anodal, cathodal or sham tDCS over the left DLPFC on the oscillatory brain activity associated with higher-order cognitive processing. Our results show that tDCS can change the organized cortical activity associated with WM in concert with systematic alterations of WM performance. To our knowledge, this is the first study investigating the effects of tDCS on oscillatory brain activity in the context of a WM task. The results of the study will provide a better understanding of the neuromodulatory effects of tDCS and demonstrate its potential both at fostering knowledge on the functional significance of brain oscillations and for therapeutic application.

\section{Methods \\ Participants}

Sixteen university students (10 females, mean age $25 \pm 2$ years) participated in the tDCS study. All participants were consistent right-handers [83] and had no metallic implant and no history of neurological or psychiatric illness. An intelligence test [84] showed that IQ levels of all the participants were in or above the range of the norm (mean $115 \pm 13$ ). Each participant gave written, informed consent prior to the experiment. All 
procedures in the study were approved by the ethics committee of the University of Zurich. In an additional control experiment a separate sample of 16 participants (12 females, mean age $24 \pm 4$ years) was investigated to measure the repetition effect on WM task performance without tDCS. This control group undertook one experimental session and performed the same behavioral task twice but without receiving any tDCS-treatment during the 15 min break in between. Experimental setting as well as instruction to the participants of the control group was identical to the stimulation group, except that no tDCS was applied and no EEG data were recorded. IQ levels of the participants in the control group were similar to those in the stimulated group (mean $117 \pm 14)$.

\section{Transcranial direct current stimulation (tDCS)}

The participants were seated comfortably in a recliner in front of a personal computer screen in an electromagnetically shielded room. TDCS was delivered by a batterydriven constant current stimulator (Eldith, NeuroCon $\mathrm{GmbH}$, Germany) using a pair of rubber electrodes in a $5 \times 7 \mathrm{~cm}$ saline-soaked synthetic sponge. For stimulation of the left DLPFC the active electrode (to which the term anodal/cathodal stimulation refers) was placed over F3 according to the International 10-20 system for electroencephalography (EEG) electrode placement [85]. The use of EEG electrode positions for DLPFC localisation has been applied before in studies using tDCS $[8,26,29,52,86]$ and transcranial magnetic stimulation (TMS) [87]. Since both the left and right prefrontal cortex have been shown to subserve the WM system $[41,88]$, and the left hemisphere seems to be particularly crucial for the processing of verbal WM tasks [42,43], we used an ipsilateral reference electrode over the left mastoid in order to avoid confounding biases arising from tDCS effects over the right hemisphere. In using this ipsilateral stimulation, we accepted the possibility that this mounting might reduce the tDCS-related effects on the underlying cortex, since it could cause current to be shunted to a greater degree through skin and cerebrospinal fluid. A constant current of $1.0 \mathrm{~mA}$ was applied for $15 \mathrm{~min}$, with a linear fade in/fade out of $10 \mathrm{~s}$. Each participant performed one anodal and one cathodal tDCS session separated by at least one day. The session order was counterbalanced across participants. Within each session, participants underwent one sham condition and one stimulation (i.e., anodal/cathodal) condition, and the sham condition always preceded the stimulation condition to avoid carry-over effects of tDCS (cf. Figure 4, top row). For the sham condition, the same electrode placement was used as in the stimulation condition, but the current was applied for $30 \mathrm{~s}$, and was then ramped down without the subject's

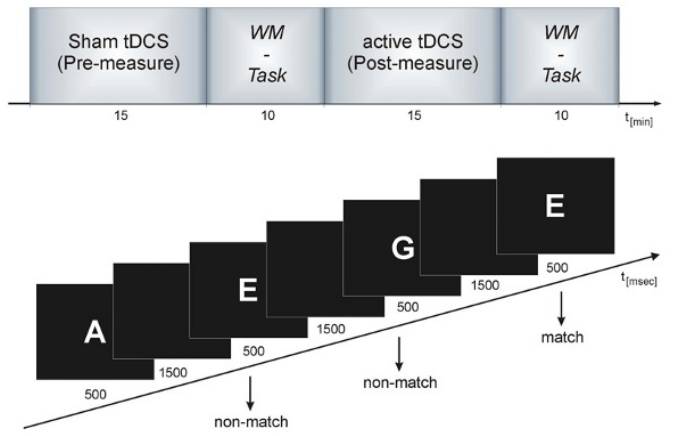

Figure 4 Experimental design. Top: After mounting EEG and tDCS electrodes, the experiment started with a 15-minute Sham-tDCS. For the next 10 minutes, participants performed a letter two-back working memory (WM) task while EEG was recorded. Subsequently, a 15 minute active-tDCS (anodal or cathodal) was applied, followed by a further 10-minute WM task. EEG recording was stopped during the stimulation periods. Each participant performed one anodal and one cathodal tDCS session separated by at least one day. The session order was counterbalanced across participants. An additional control group underwent one experimental session performing the similar WM task twice but without receiving a treatment during the 15 minute break in between. Bottom: Schematic description of the letter two-back working memory task.

awareness. This procedure ensured that in both the sham and stimulation condition, participants experienced the initial itching that recedes over the first seconds of tDCS [89]. Accordingly, none of the participants was able to determine whether or not they received real or sham stimulation.

\section{Working memory assessment}

After each sham and stimulation condition, participants performed a two-back letter working memory (WM) task with concurrent EEG recording. Stimulus presentation was controlled by the Presentation software (Neurobehavioral Systems, USA). Participants were stimulated with a sequence of white letters (A, B, C, D, E) which were presented on a black background in the centre of the screen. Each letter was presented for $500 \mathrm{~ms}$ with an interstimulus interval of $2 \mathrm{~s}$. Each letter was followed by a white fixation cross that remained until the next letter was presented. A target letter was any letter repeated after one intervening letter. Participants were asked to respond to each letter as quickly and accurately as possible and to indicate whether the currently presented letter matched (left button) or did not match (right button) the letter which was presented two trials before (cf. Figure 4, bottom row). A brief practice sequence of 100 trials was given before the actual test. The test sequence consisted of 300 letters, with 77 matching letters (targets) and 223 non-matching letters (rejections). 


\section{EEG recording and preprocessing}

During the WM task, EEG was recorded continuously with thirty-two electrodes (Fp1, Fp2, F7, F3, Fz, F4, F8, FT7, FC3, FCz, FC4, FT8, T7, C3, Cz, C4, T8, TP9, TP7, CP3, CPz, CP4, TP8, TP10, P7, P3, Pz, P4, P8, O1, $\mathrm{Oz}, \mathrm{O} 2)$ located according to the International 10-20 system [85]. The electro-oculogram was recorded from two bipolar electrode pairs placed above and below the left eye, and on the outer canthi of each eye, respectively. A QuickAmp amplifier system (BrainProducts, Munich, Germany) was used for EEG recording. EEG data were recorded against the grand average reference and sampled at $500 \mathrm{~Hz}$, and impedances were kept below $10 \mathrm{k} \Omega$. EEG preprocessing and data analysis were carried out in Brain Vision Analyzer 2.0 (BrainProducts, Munich, Germany), EEGLAB 6.01 [90] and FieldTrip http://fieldtrip.fcdonders.nl/. EEG data were off-line treated with a $24 \mathrm{~dB}$ zero-phase Butterworth filter from 0.1 to $30 \mathrm{~Hz}$ and were segmented into epochs from -312 to 712 ms relative to stimulus onset. After baseline correction ( -312 to $0 \mathrm{~ms})$, epochs were automatically screened for unique and non-stereotyped artifacts using a probability function built into EEGLAB [91]. With this procedure, epochs that contained signal values exceeding three standard deviations were removed. Independent component analysis (ICA) was then applied to remove ocular artifacts [92,93]. After artifact removal, averages were computed for all remaining correct target responses (i.e., hits) for each of the four conditions (anodal, cathodal, sham preceding anodal (shamA), sham preceding cathodal (shamC)). In order to avoid multiple comparisons between neighbouring electrodes and to increase the signal-to-noise ratio, data from selected electrode sites (Pz, P3, P4, Oz, O1, O2) were pooled into an occipito-parietal ROI.

\section{Data Analysis \\ Behavioural data}

To evaluate performance in the context of signal detection theory, d' was analyzed. As recently demonstrated, d' has the advantage to capture executive skills needed to perform n-back WM tasks without being influenced by demographic variables or IQ [94]. Values for d' were estimated for each subject by dividing the difference of $\mathrm{Z}$ (false alarms) and $\mathrm{Z}$ (hits) by the root mean square of 2 . The discriminability measure d' captures the ability of the participant to discriminate between the two stimulus types, here the two-back target letters and the non-target letters. Additionally, reaction times (RT) for Hits and False Alarms (FA) were analyzed. To evaluate the impact of tDCS on the repetition-related increase in WM performance, we analyzed the pre-to-post measurements by computing the difference between the behavioural values assessed after active stimulation (anodal/ cathodal) and the preceding sham stimulation (shamA/ sham $C$ ), separately for the anodal (delta anodal, $\Delta \mathrm{A}$ ) and cathodal session (delta cathodal, $\Delta \mathrm{C}$ ). We compared these pre-to-post measurements, $\Delta \mathrm{A}$ and $\Delta \mathrm{C}$, for each behavioural parameter using paired t-tests. Prior to this calculation, we evaluated the equality of the two sham conditions using paired t-test. This statistical analysis revealed no significant difference between the two sham conditions for d' $\left(t_{15}=-0.34, P=0.7\right)$. Similarly, RTs for Hits $\left(t_{15}=0.74, P=0.5\right)$ and False Alarms $\left(t_{15}=0.27, P\right.$ $=0.9$ ) were not significantly different between the two sham conditions. To determine tDCS-induced modulations on the repetition-related increase in WM performance, we compared the pre-post differences $(\Delta \mathrm{A}$ and $\Delta C$ ) of the tDCS-treated group with pre-post measurements of a separate control group $(\Delta C G)$ that also performed the WM task twice but without receiving a tDCS treatment during the break in between ( $\Delta \mathrm{A}$ vs. $\Delta C G$, and $\Delta C$ vs. $\Delta C G$ ). Again, prior to this calculation, we evaluated the equality of the two experimental sham conditions of the tDCS-treated group and the pre-measurement (i.e., first repetition of the WM task) in the control group by means of independent-samples t-tests. This statistical analysis revealed no significant differences between the sham conditions of the tDCS-treated group and the pre-measurement of the control group (cf. Table 2).

\section{Analysis of event-related potentials}

Event-related potentials (ERPs) were measured relative to the pre-stimulus baseline $(-312-0 \mathrm{~ms})$ for each condition (anodal, cathodal, sham preceding anodal (shamA), sham preceding cathodal (shamC)). Peak detection was performed for the occipito-parietal ROI (P1, N1 component) and for the Pz electrode (P3 component). This procedure entailed the detection of the most positive (P1, P3 component) or negative (N1 component) peaks within specific latency bands (P1: 80-140 ms; N1: 120-250 ms; P3: 230-500 ms). The time

\section{Table 2 Comparison of pre-measurements}

\begin{tabular}{lllll}
\hline condition & measure & $\boldsymbol{t}$ & $\mathbf{d f}$ & $\boldsymbol{P}$ \\
\hline sham A vs. CG & & & & \\
& d' & 0.687 & 30 & 0.500 \\
& RT-Hits & -0.910 & 30 & 0.371 \\
& RT-FA & -0.528 & 30 & 0.602 \\
sham C vs. CG & & & & \\
& d' & 1.080 & & 0.290 \\
& RT-Hits & -1.484 & 30 & 0.148 \\
& RT-FA & -0.694 & 30 & 0.493
\end{tabular}

Table shows results of independent t-tests comparing the pre-measurements for d', RT-Hits (reaction time for Hits), and RT-FA (reaction time for False Alarms). Results show equivalence of pre-measures between the two experimental groups (shamA: sham condition preceding the anodal tDCS, shamC: sham condition preceding the cathodal tDCS) and control group (CG). 
windows for peak analysis were defined on the basis of the global field power.

\section{Analysis of event-related spectral perturbation}

For each subject and each channel, event-related spectral perturbation (ERSP) was calculated using a waveletbased analysis implemented in Brain Vision Analyzer 2.0 software. We used a continuous wavelet transform (WT) with complex Morlet wavelets (morlet parameter c 3.8; 30 frequency steps from 1 to $30 \mathrm{~Hz}$ ) to examine the frequency composition of single-trial epochs. The magnitudes of the WTs of single-trial epochs were then averaged to compute the total power of activity, which contains signal components that are phase-locked and non-phase-locked to the stimulus event. For each scale of the WT a baseline correction was applied by subtracting the mean amplitude within the -200 to $-100 \mathrm{~ms}$ time window from each data point after stimulus onset. Similar to the procedures used in the ERP analysis, the data were subsequently pooled into an occipito-parietal ROI by averaging the ERSPs across different electrode sites (Pz, P3, P4, Oz, O1, O2).

TDCS effects on oscillatory brain activity were analyzed by computing ERSP differences between the separate conditions. For statistical comparisons, we used a nonparametric cluster-based randomization approach built into FieldTrip. This procedure defined clusters on the basis of the actual distribution of the data and tested the statistical significance of these clusters using a Monte-Carlo randomization method with correction for multiple comparisons [95]. The clustering used 500 randomizations and was performed in time and frequency simultaneously. The t-statistic of paired t-tests was calculated on a cluster-level by taking the sum of the tvalues within the respective cluster.

\section{Acknowledgements}

This study was supported by the Deutsche Forschungsgemeinschaft (SFB/ TR31-TPA9) (TZ, CSH) and the Swiss National Foundation (PBZHP3-128462) (PS).

\section{Author details \\ 'Department of Neurology, Otto v. Guericke University Magdeburg, Germany. ${ }^{2}$ German Centre for Neurodegenerative Diseases (DZNE) Magdeburg, Germany. ${ }^{3}$ Department of Psychology, Neuropsychology Lab, Carl von Ossietzky University of Oldenburg, Germany. ${ }^{4}$ Institute of Psychology, Division of Neuropsychology, University of Zurich, Switzerland. ${ }^{5}$ Department of Experimental Psychology, Carl von Ossietzky University Oldenburg, Germany.}

\section{Authors' contributions}

TZ: conceived of the study, designed the experimental paradigm, performed the statistical analysis and drafted the manuscript

PS: conceived of the study, performed the data acquisition and the statistical analysis and drafted the manuscript

JDT: performed statistical analysis and contributed to the manuscript LJ: contributed to the hypothesis, and to the preparation of the manuscript CSH: contributed to the design, discussion, and to the preparation of the manuscript

All authors read and approved the final manuscript.
Received: 1 October 2010 Accepted: 6 January 2011

Published: 6 January 2011

\section{References}

1. Nitsche MA, Cohen LG, Wassermann EM, Priori A, Lang N, Antal A Paulus W, Hummel F, Boggio PS, Fregni F, Pascual-Leone A: Transcranial direct current stimulation: State of the art 2008. Brain Stimulation 2008, 1:206-223.

2. Miranda PC, Lomarev M, Hallett M: Modeling the current distribution during transcranial direct current stimulation. Clinical Neurophysiology 2006, 117:1623-1629.

3. Nitsche MA, Nitsche MS, Klein CC, Tergau F, Rothwell JC, Paulus W: Level of action of cathodal DC polarisation induced inhibition of the human motor cortex. Clinical Neurophysiology 2003, 114:600-604

4. Nitsche MA, Paulus W: Excitability changes induced in the human motor cortex by weak transcranial direct current stimulation. The Journal of Physiology 2000, 527:633-639.

5. Ardolino G, Bossi B, Barbieri S, Priori A: Non-synaptic mechanisms underlie the after-effects of cathodal transcutaneous direct current stimulation of the human brain. The Journal of Physiology 2005, 568:653-663.

6. Bindman $L$, Lippold OC, Redfearn JW: Long-lasting changes in the level of the electrical activity of the cerebral cortex produced bypolarizing currents. Nature 1962, 196:584-585

7. Purpura DP, McMurtry JG: Intracellular activities and evoked potential changes during polarization of motor cortex. Journal of Neurophysiology 1965, 28:166-185.

8. Ohn SH, Park Cl, Yoo WK, Ko MH, Choi KP, Kim GM, Lee YT, Kim YH: Timedependent effect of transcranial direct current stimulation on the enhancement of working memory. Neuroreport 2008, 19:43-47.

9. Gartside IB: Mechanisms of sustained increases of firing rate of neurons in the rat cerebral cortex after polarization: reverberating circuits or modification of synaptic conductance? Nature 1968, 220:382-383.

10. Hattori $Y$, Moriwaki A, Hori Y: Biphasic effects of polarizing current on adenosine-sensitive generation of cyclic AMP in rat cerebral cortex. Neuroscience Letters 1990, 116:320-324.

11. Islam N, Aftabuddin M, Moriwaki A, Hattori $Y$, Hori $Y$ : Increase in the calcium level following anodal polarization in the rat brain. Brain Research 1995, 684:206-208.

12. Liebetanz D, Nitsche MA, Tergau F, Paulus W: Pharmacological approach to the mechanisms of transcranial DC-stimulation-induced after-effects of human motor cortex excitability. Brain 2002, 125:2238-2247.

13. Nitsche MA, Jaussi W, Liebetanz D, Lang N, Tergau F, Paulus W: Consolidation of human motor cortical neuroplasticity by D-cycloserine. Neuropsychopharmacology 2004, 29:1573-1578.

14. Wagner T, Valero-Cabre A, Pascual-Leone A: Noninvasive human brain stimulation. Annual Review of Biomedical Engineering 2007 9:527-565.

15. Morrell F, Naitoh P: Effect of cortical polarization on a conditioned avoidance response. Experimental Neurology 1962, 6:507-532.

16. Yamaguchi $K$, Hori $Y$ : Long lasting retention of cortical dominant focus in rabbit. Medical Journal of Osaka University 1975, 26:39-50.

17. Rosen SC, Stamm JS: Transcortical polarization: facilitation of delayed response performance by monkeys. Experimental Neurology 1972 , 35:282-289.

18. Been G, Ngo $T$, Miller SM, Fitzgerald PB: The use of tDCS and CVS as methods of non-invasive brain stimulation. Brain Research Reviews 2007, 56:346-361.

19. Nitsche MA, Schauenburg A, Lang N, Liebetanz D, Exner C, Paulus W, Tergau F: Facilitation of implicit motor learning by weak transcranial direct current stimulation of the primary motor cortex in the human. Journal of Cognitive Neuroscience 2003, 15:619-626.

20. Vines BW, Cerruti C, Schlaug G: Dual-hemisphere tDCS facilitates greater improvements for healthy subjects' non-dominant hand compared to uni-hemisphere stimulation. BMC Neuroscience 2008, 9:103.

21. Antal A, Nitsche MA, Kincses TZ, Kruse W, Hoffmann KP, Paulus W: Facilitation of visuo-motor learning by transcranial direct current stimulation of the motor and extrastriate visual areas in humans. The European Journal of Neuroscience 2004, 19:2888-2892.

22. Vines BW, Schnider NM, Schlaug G: Testing for causality with transcranial direct current stimulation: pitch memory and the left supramarginal gyrus. Neuroreport 2006, 17:1047-1050. 
23. Flöel A, Rösser N, Michka O, Knecht S, Breitenstein C: Noninvasive brain stimulation improves language learning. Journal of Cognitive Neuroscience 2008, 20:1415-22.

24. Fiori V, Coccia M, Marinelli CV, Vecchi V, Bonifazi S, Ceravolo MG, Provinciali L, Tomaiuolo F, Marangolo P: Transcranial Direct Current Stimulation Improves Word Retrieval in Healthy and Nonfluent Aphasic Subjects. Journal of Cognitive Neuroscience 2010.

25. de Vries MH, Barth AC, Maiworm S, Knecht S, Zwitserlood P, Flöel A: Electrical stimulation of Broca's area enhances implicit learning of an artificial grammar. Journal of Cognitive Neuroscience 2010, 22:2427-36.

26. Elmer S, Burkard M, Renz B, Meyer M, Jancke L: Direct current induced short-term modulation of the left dorsolateral prefrontal cortex while learning auditory presented nouns. Behavioral and Brain Functions 2009, 5:29.

27. Liuzzi G, Freundlieb N, Ridder V, Hoppe J, Heise K, Zimerman M, Dobel C, Enriquez-Geppert S, Gerloff C, Zwitserlood P, Hummel FC: The involvement of the left motor cortex in learning of a novel action word lexicon. Current Biology 2010, 20:1745-51.

28. Boggio PS, Ferrucci R, Rigonatti SP, Covre P, Nitsche M, Pascual-Leone A, Fregni F: Effects of transcranial direct current stimulation on working memory in patients with Parkinson's disease. Journal of the Neurological Sciences 2006, 31-38.

29. Fregni $F$, Boggio PS, Nitsche M, Bermpohl F, Antal A, Feredoes $E$, Marcolin MA, Rigonatti SP, Silva MT, Paulus W, Pascual-Leone A: Anodal transcranial direct current stimulation of prefrontal cortex enhances working memory. Experimental Brain Research 2005, 166:23-30.

30. Baddeley A: Working memory. Science 1992, 255:556-559.

31. Fuster JM: The prefrontal cortex, mediator of cross-temporal contingencies. Human Neurobiology 1985, 4:169-179.

32. Goldman-Rakic PS: Development of cortical circuitry and cognitive function. Child Development 1987, 58:601-622.

33. Passingham D, Sakai K: The prefrontal cortex and working memory: physiology and brain imaging. Current Opinion in Neurobiology 2004 14:163-168.

34. Courtney SM, Petit L, Haxby JV, Ungerleider LG: The role of prefrontal cortex in working memory: examining the contents of consciousness. Philosophical Transactions of the Royal Society of London 1998, 353:1819-1828

35. Hautzel H, Mottaghy FM, Schmidt D, Zemb M, Shah NJ, Muller-Gartner HW, Krause BJ: Topographic segregation and convergence of verbal, object, shape and spatial working memory in humans. Neuroscience Letters 2002, 323:156-160

36. Nystrom LE, Braver TS, Sabb FW, Delgado MR, Noll DC, Cohen JD: Working memory for letters, shapes, and locations: fMRI evidence against stimulus-based regional organization in human prefrontal cortex. Neuroimage 2000, 11:424-446

37. Smith EE, Jonides J: Working memory: a view from neuroimaging Cognitive Psychology 1997, 33:5-42.

38. Owen AM, McMillan KM, Laird AR, Bullmore E: N-back working memory paradigm: a meta-analysis of normative functional neuroimaging studies. Human Brain Mapping 2005, 25:46-59.

39. Funahashi S, Bruce CJ, Goldman-Rakic PS: Mnemonic coding of visua space in the monkey's dorsolateral prefrontal cortex. Journal of Neurophysiology 1989, 61:331-349.

40. Callicott JH, Mattay VS, Bertolino A, Finn K, Coppola R, Frank JA, Goldberg TE, Weinberger DR: Physiological characteristics of capacity constraints in working memory as revealed by functional MRI. Cerebral Cortex 1999, 9:20-26.

41. D'Esposito M, Aguirre GK, Zarahn E, Ballard D, Shin RK, Lease J: Functional MRI studies of spatial and nonspatial working memory. Brain Research 1998, 7:1-13.

42. Tsuchida A, Fellows LK: Lesion evidence that two distinct regions within prefrontal cortex are critical for n-back performance in humans. Journal of Cognitive Neuroscience 2009, 21:2263-2275.

43. Mull BR, Seyal M: Transcranial magnetic stimulation of left prefrontal cortex impairs working memory. Clinical Neurophysiology 2001, 112:1672-1675

44. Klimesch W, Schack B, Sauseng P: The functional significance of theta and upper alpha oscillations. Experimental Psychology 2005, 52:99-108.

45. Boggio PS, Rigonatti SP, Ribeiro RB, Myczkowski ML, Nitsche MA, PascualLeone A, Fregni F: A randomized, double-blind clinical trial on the efficacy of cortical direct current stimulation for the treatment of major depression. The International Journal of Neuropsychopharmacology 2008, 11:249-254.

46. Ferrucci R, Bortolomasi M, Vergari M, Tadini L, Salvoro B, Giacopuzzi M, Barbieri S, Priori A: Transcranial direct current stimulation in severe, drug-resistant major depression. Journal of Affective Disorders 2009, 118:215-219

47. Hummel FC, Voller B, Celnik P, Floel A, Giraux P, Gerloff C, Cohen LG: Effects of brain polarization on reaction times and pinch force in chronic stroke. BMC Neuroscience 2006, 7:73.

48. Monti A, Cogiamanian F, Marceglia S, Ferrucci R, Mameli F, Mrakic-Sposta S, Vergari $M$, Zago S, Priori A: Improved naming after transcranial direct current stimulation in aphasia. Journal of Neurology, Neurosurgery, and Psychiatry 2008, 79:451-453.

49. Nitsche MA, Boggio PS, Fregni F, Pascual-Leone A: Treatment of depression with transcranial direct current stimulation (tDCS): a review. Experimental Neurology 2009, 219:14-19.

50. Schlaug $G$, Renga $V$ : Transcranial direct current stimulation: a noninvasive tool to facilitate stroke recovery. Expert Review of Medical Devices 2008, 5:759-768

51. Schlaug G, Renga V, Nair D: Transcranial direct current stimulation in stroke recovery. Archives of Neurology 2008, 65:1571-1576.

52. Jo JM, Kim YH, Ko MH, Ohn SH, Joen B, Lee KH: Enhancing the working memory of stroke patients using tDCS. American Journal of Physical Medicine \& Rehabilitation 2009, 88:404-409.

53. Boggio PS, Khoury LP, Martins DC, Martins OE, de Macedo EC, Fregni F: Temporal cortex direct current stimulation enhances performance on a visual recognition memory task in Alzheimer disease. Journal of Neurology, Neurosurgery, and Psychiatry 2009, 80:444-447.

54. Ferrucci R, Mameli F, Guidi I, Mrakic-Sposta S, Vergari M, Marceglia S, Cogiamanian F, Barbieri S, Scarpini E, Priori A: Transcranial direct current stimulation improves recognition memory in Alzheimer disease. Neurology 2008, 71:493-498.

55. Marshall L, Molle M, Siebner HR, Born J: Bifrontal transcranial direct current stimulation slows reaction time in a working memory task. $B M C$ Neuroscience 2005, 6:23.

56. Miranda PC, Faria P, Hallett M: What does the ratio of injected current to electrode area tell us about current density in the brain during tDCS. Clinical Neurophysiology 2009, 120:1183-1187.

57. Datta A, Bansal V, Diaz J, Patel J, Reato D, Bikson M: Gyri-precise head model of transcranial direct current stimulation: Improved spatial focality using a ring electrode versus conventional rectangular pad. Brain Stimulation 2009, 2:201-207.

58. Antal A, Kincses TZ, Nitsche MA, Bartfai O, Paulus W: Excitability changes induced in the human primary visual cortex by transcranial direct current stimulation: direct electrophysiological evidence. Investigative Ophthalmology \& Visual Science 2004, 45:702-707.

59. Accornero N, Li Voti P, La Riccia M, Gregori B: Visual evoked potentials modulation during direct current cortical polarization. Experimental Brain Research 2007, 178:261-266.

60. Antal A, Brepohl N, Poreisz C, Boros K, Csifcsak G, Paulus W: Transcrania direct current stimulation over somatosensory cortex decreases experimentally induced acute pain perception. The Clinical Journal of Pain 2008, 24:56-63.

61. Dieckhofer A, Waberski TD, Nitsche M, Paulus W, Buchner H, Gobbele R: Transcranial direct current stimulation applied over the somatosensory cortex - differential effect on low and high frequency SEPs. Clinical Neurophysiology 2006, 117:2221-2227.

62. Matsunaga K, Nitsche MA, Tsuji S, Rothwell JC: Effect of transcranial DC sensorimotor cortex stimulation on somatosensory evoked potentials in humans. Clinical Neurophysiology 2004, 115:456-460.

63. Nitsche MA, Paulus W: Sustained excitability elevations induced by transcranial DC motor cortex stimulation in humans. Neurology 2001, 57:1899-1901.

64. Antal A, Varga ET, Kincses TZ, Nitsche MA, Paulus W: Oscillatory brain activity and transcranial direct current stimulation in humans. Neuroreport 2004, 15:1307-1310.

65. Sparing R, Mottaghy FM: Noninvasive brain stimulation with transcranial magnetic or direct current stimulation (TMS/tDCS)-From insights into human memory to therapy of its dysfunction. Methods 2008, 44:329-337. 
66. Herrmann CS, Frund I, Lenz D: Human gamma-band activity: a review on cognitive and behavioral correlates and network models. Neuroscience and Biobehavioral Reviews 2010, 34:981-992.

67. Herrmann CS, Munk MH, Engel AK: Cognitive functions of gamma-band activity: memory match and utilization. Trends in Cognitive Sciences 2004 8:347-355

68. Buzsaki G: Rhythms of the brain Oxford: Oxford University Press; 2006.

69. Lisman JE, Idiart MA: Storage of $7+/-2$ short-term memories in oscillatory subcycles. Science 1995, 267:1512-1515.

70. Pesonen $\mathrm{M}$, Hamalainen $\mathrm{H}$, Krause $\mathrm{CM}$ : Brain oscillatory $4-30 \mathrm{~Hz}$ responses during a visual $n$-back memory task with varying memory load. Brain Research 2007, 1138:171-177.

71. Klimesch W, Doppelmayr M, Rohm D, Pollhuber D, Stadler W: Simultaneous desynchronization and synchronization of different alpha responses in the human electroencephalograph: a neglected paradox. Neuroscience Letters 2000, 284:97-100.

72. Klimesch W, Doppelmayr M, Schwaiger J, Auinger P, Winkler T: 'Paradoxical' alpha synchronization in a memory task. Brain Research 1999, 7:493-501.

73. Cooper NR, Croft RJ, Dominey SJ, Burgess AP, Gruzelier JH: Paradox lost? Exploring the role of alpha oscillations during externally vs. internally directed attention and the implications for idling and inhibition hypotheses. International Journal of Psychophysiology 2003, 47:65-74.

74. Jensen $\mathrm{O}$, Tesche CD: Frontal theta activity in humans increases with memory load in a working memory task. The European Journal of Neuroscience 2002, 15:1395-1399.

75. Karrasch M, Laine M, Rapinoja P, Krause CM: Effects of normal aging on event-related desynchronization/synchronization during a memory task in humans. Neuroscience Letters 2004, 366:18-23.

76. Klimesch W, Doppelmayr M, Stadler W, Pollhuber D, Sauseng P, Rohm D: Episodic retrieval is reflected by a process specific increase in human electroencephalographic theta activity. Neuroscience Letters 2001, 302:49-52.

77. Pesonen $\mathrm{M}$, Bjornberg $\mathrm{CH}$, Hamalainen $\mathrm{H}$, Krause CM: Brain oscillatory 1-30 $\mathrm{Hz}$ EEG ERD/ERS responses during the different stages of an auditory memory search task. Neuroscience Letters 2006, 399:45-50.

78. Baddeley A: Modularity, mass-action and memory. The Quarterly Journal of Experimental Psychology 1986, 38:527-533.

79. Baddeley A: Working memory: looking back and looking forward. Nature Reviews 2003, 4:829-839.

80. Sauseng P, Klimesch W, Schabus M, Doppelmayr M: Fronto-parietal EEG coherence in theta and upper alpha reflect central executive functions of working memory. International Journal of Psychophysiology 2005, 57:97-103

81. Gevins A, Smith ME, McEvoy L, Yu D: High-resolution EEG mapping of cortical activation related to working memory: effects of task difficulty, type of processing, and practice. Cerebral Cortex 1997, 7:374-385.

82. Lang N, Siebner HR, Ward NS, Lee L, Nitsche MA, Paulus W, Rothwell JC, Lemon RN, Frackowiak RS: How does transcranial DC stimulation of the primary motor cortex alter regional neuronal activity in the human brain. The European Journal of Neuroscience 2005, 22:495-504.

83. Annett M: A classification of hand preference by association analysis. British Journal of Psychology 1970, 61:303-321.

84. Lehrl S, Gallwitz A, Blaha L: Kurztest für Allgemeine Intelligenz Göttingen: Hogrefe Testznetrale; 1992.

85. Jasper $\mathrm{HH}$ : The ten-twenty electrode system of the International Federation. Electroencephalography and Clinical Neurophysiology 1958, 10:371-375.

86. Beeli G, Casutt G, Baumgartner T, Jancke L: Modulating presence and impulsiveness by external stimulation of the brain. Behavioral and Brain Functions 2008, 4:33

87. Rossi S, Cappa SF, Babiloni C, Pasqualetti P, Miniussi C, Carducci F, Babiloni F, Rossini PM: Prefrontal [correction of Prefontal] cortex in longterm memory: an "interference" approach using magnetic stimulation. Nature Neuroscience 2001, 4:948-952.

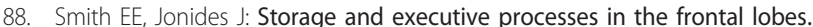
Science 1999, 283:1657-61.

89. Gandiga PC, Hummel FC, Cohen LG: Transcranial DC stimulation (tDCS): a tool for double-blind sham-controlled clinical studies in brain stimulation. Clinical Neurophysiology 2006, 117:845-850
90. Delorme A, Makeig S: EEGLAB: an open source toolbox for analysis of single-trial EEG dynamics including independent component analysis. Journal of Neuroscience Methods 2004, 134:9-21.

91. Delorme A, Sejnowski T, Makeig S: Enhanced detection of artifacts in EEG data using higher-order statistics and independent component analysis. Neuroimage 2007, 34:1443-1449.

92. Jung TP, Makeig S, Humphries C, Lee TW, McKeown MJ, Iragui V, Sejnowski TJ: Removing electroencephalographic artifacts by blind source separation. Psychophysiology 2000, 37:163-178.

93. Jung TP, Makeig S, Westerfield M, Townsend J, Courchesne E, Sejnowski TJ: Removal of eye activity artifacts from visual event-related potentials in normal and clinical subjects. Clinical Neurophysiology 2000, 111:1745-1758.

94. Haatveit BC, Sundet K, Hugdahl K, Ueland T, Melle I, Andreassen OA: The validity of $d$ prime as a working memory index: Results from the "Bergen n-back task". Journal of Clinical and Experimental Neuropsychology 1-10.

95. Maris E, Oostenveld R: Nonparametric statistical testing of EEG- and MEGdata. Journal of Neuroscience Methods 164:177-90.

doi:10.1186/1471-2202-12-2

Cite this article as: Zaehle et al: Transcranial direct current stimulation of the prefrontal cortex modulates working memory performance: combined behavioural and electrophysiological evidence. BMC Neuroscience 2011 12:2.

\section{Submit your next manuscript to BioMed Central and take full advantage of:}

- Convenient online submission

- Thorough peer review

- No space constraints or color figure charges

- Immediate publication on acceptance

- Inclusion in PubMed, CAS, Scopus and Google Scholar

- Research which is freely available for redistribution

Submit your manuscript at www.biomedcentral.com/submit
C) Biomed Central 